\title{
Video Article \\ RNAscope for In situ Detection of Transcriptionally Active Human Papillomavirus in Head and Neck Squamous Cell Carcinoma
}

\author{
Hongwei Wang ${ }^{1}$, Mindy Xiao-Ming Wang ${ }^{1}$, Nan Su${ }^{1}$, Li-chong Wang ${ }^{1}$, Xingyong Wu ${ }^{1}$, Son Bui ${ }^{1}$, Allissa Nielsen ${ }^{1}$, Hong-Thuy Vo ${ }^{1}$, Nina Nguyen ${ }^{1}$, \\ Yuling Luo ${ }^{1}$, Xiao-Jun Ma ${ }^{1}$ \\ ${ }^{1}$ Advanced Cell Diagnostics, Inc.
}

Correspondence to: Xiao-Jun Ma at xma@acdbio.com

URL: https://www.jove.com/video/51426

DOI: doi: $10.3791 / 51426$

Keywords: Medicine, Issue 85, RNAscope, Head and Neck Squamous Cell Carcinoma (HNSCC), Oropharyngeal Squamous Cell Carcinoma (OPSCC), Human Papillomavirus (HPV), E6/ E7 mRNA, in situ hybridization, tumor

Date Published: 3/11/2014

Citation: Wang, H., Wang, M.X., Su, N., Wang, L.c., Wu, X., Bui, S., Nielsen, A., Vo, H.T., Nguyen, N., Luo, Y., Ma, X.J. RNAscope for In situ Detection of Transcriptionally Active Human Papillomavirus in Head and Neck Squamous Cell Carcinoma. J. Vis. Exp. (85), e51426, doi:10.3791/51426 (2014).

\section{Abstract}

The 'gold standard' for oncogenic HPV detection is the demonstration of transcriptionally active high-risk HPV in tumor tissue. However, detection of E6/E7 mRNA by quantitative reverse transcription polymerase chain reaction (qRT-PCR) requires RNA extraction which destroys the tumor tissue context critical for morphological correlation and has been difficult to be adopted in routine clinical practice. Our recently developed RNA in situ hybridization technology, RNAscope, permits direct visualization of RNA in formalin-fixed, paraffin-embedded (FFPE) tissue with single molecule sensitivity and single cell resolution, which enables highly sensitive and specific in situ analysis of any RNA biomarker in routine clinical specimens. The RNAscope HPV assay was designed to detect the E6/E7 mRNA of seven high-risk HPV genotypes (HPV16, 18, 31, 33, 35, 52 , and 58) using a pool of genotype-specific probes. It has demonstrated excellent sensitivity and specificity against the current 'gold standard' method of detecting E6/E7 mRNA by qRT-PCR. HPV status determined by RNAscope is strongly prognostic of clinical outcome in oropharyngeal cancer patients.

\section{Video Link}

The video component of this article can be found at https://www.jove.com/video/51426/

\section{Introduction}

High-risk human papillomavirus (HR-HPV) infection accounts for approximately $5 \%$ of all cancers worldwide ${ }^{1}$. The incidence of HPV-associated oropharyngeal cancer has increased during the past decades, especially among men. HPV-positive oropharyngeal squamous cell carcinoma (OPSCC) will likely constitute a majority of all head and neck cancers in the United States in the next 20 years ${ }^{2}$. Oropharyngeal squamous cell carcinomas caused by HPV are associated with favorable survival, and tumor HPV status is a strong and independent prognostic factor for survival $^{3}$.

Evidence for transcriptional activation of the viral oncogenes E6 and E7 is regarded as the gold standard for the presence of clinically relevant $\mathrm{HPV}^{4}$. However, detection of E6/E7 mRNA from fresh tumor tissue by RT-PCR technique is not practical in routine clinical practice and has been limited to the research laboratories. Recently, we have developed a novel RNA ISH technology called RNAscope, which enables multiplex detection in individual cells with single RNA molecule sensitivity in formalin fixed paraffin embedded tissue specimens (FFPE) ${ }^{5-10}$. We provided three lines of evidence for single molecule detection ${ }^{5}$. First, the RNAscope probe design and signal amplification system allowed detection of single copy HER2 genomic DNA targets in HeLa and SK-BR-3 cell lines. Second, when compared with HER2 genomic DNA signals, the distribution of the fluorescent intensities of HER2 mRNA signal dots in HeLa cells was consistent with one molecule per dot. Thirdly, the number of HER2 mRNA signal dots per cell matched closely the HER2 mRNA copy number estimated by a solution-based quantification assay, further supporting single molecule detection. Furthermore, counterstaining of nuclei with DAPI in fluorescent microscopy or with hematoxylin in brightfield microscopy allows visualization of individual nuclei, which in turn allows detection and quantification of RNA targets on a single-cell basis ${ }^{10}$ The ability to analyze gene expression in situ in routine clinical specimens makes RNAscope a promising platform for diagnostic pathology, especially those FFPE tissue section-based assays ${ }^{10,11}$. We have developed an RNAscope-based HPV assay to detect E6/E7 mRNA of seven high-risk HPV genotypes (HPV16, 18, 31, 33, 35, 52, and 58) using a pool of genotype-specific probes. Our recent studies in OPSCC have shown that the RNAscope HPV assay is highly sensitive and specific in determining HPV status on FFPE tissues ${ }^{12-17}$, and also informs prognosis in OPSCC ${ }^{12,16}$.

The principle of the RNAscope technology has been previously described ${ }^{5}$. Here, we describe the complete RNAscope assay protocol and demonstrate its use in the detection of HR-HPV in FFPE tumor tissue sections. 


\section{Protocol}

\section{Sample, Equipment, and Reagent Preparation}

1. Cut tissue specimen into blocks of 3-4 mm in thickness, fix in fresh $10 \%$ neutral-buffered formalin for $16-32 \mathrm{hr}$ at room temperature $\left(25{ }^{\circ} \mathrm{C}\right)$ and embed in paraffin. Cut FFPE into sections of $5 \pm 1 \mu \mathrm{m}$ thickness from FFPE blocks, mount sections on slides and air dry. Note: The slides can be stored at room temperature under desiccation for up to 3 months. The mounted tissue slides should be baked in a dry over at $60{ }^{\circ} \mathrm{C}$ for $1 \mathrm{hr}$ prior to the RNAscope assay.

2. Bring Hybridization Oven to $40^{\circ} \mathrm{C}$. Place a Humidifying Paper in the Humidity Control Tray. Add $50 \mathrm{ml} \mathrm{dH}_{2} \mathrm{O}$ to the Humidifying Paper to wet it completely. Insert the covered Tray into the Oven to prewarm for at least $30 \mathrm{~min}$ before use.

3. Make $700 \mathrm{ml} 1 \times$ Pretreat 2 Solution ( $10 \mathrm{nl}, \mathrm{pH} 6$ citrate buffer) by diluting $10 x$ Pretreatment Solution in $\mathrm{dH}_{2} \mathrm{O}$. Heat the $1 \times$ Pretreat 2 Solution to boil and maintain the temperature between $100-104{ }^{\circ} \mathrm{C}$ while preventing over-boiling.

4. Make $3 \mathrm{~L} 1 \times$ Wash Buffer $(0.1 \times \mathrm{SSC})$ by diluting prewarmed $50 \times$ Wash Buffer in $\mathrm{dH}_{2} \mathrm{O}$.

5. Prepare $2 \times 200 \mathrm{ml}$ of xylene and $2 \times 200 \mathrm{ml}$ of $100 \% \mathrm{EtOH}$ for deparaffinization under a fume hood.

6. Prepare $50 \%$ Hematoxylin staining solution and bluing reagent $(0.01 \%$ ammonia water $)$ for post-staining under a fume hood.

7. Prepare $200 \mathrm{ml}$ of xylene, $200 \mathrm{ml}$ of $70 \%$, and $2 \times 200 \mathrm{ml}$ of $100 \% \mathrm{EtOH}$ for dehydration under a fume hood.

8. Prewarm Target probes at $40^{\circ} \mathrm{C}$ for $10 \mathrm{~min}$ and bring RTU Detection Kit reagent to room temperature, including RTU Amp1, Amp2, Amp3, Amp4, Amp 5-Brown, and Amp6-Brown, except DAB Chromogen Brown-A and Brown-B.

\section{RNAscope Assay}

\section{Deparaffinization and Dehydration}

After baking, deparaffinize tissue sections in xylene for $2 \times 5$ min with frequent agitation, and dehydrate in $100 \%$ EtOH for $2 \times 3$ min with frequent agitation. Air dry for $5 \mathrm{~min}$ and draw a hydrophobic barrier around the tissue section with a Hydrophobic Barrier Pen.

\section{Pretreatments}

1. Incubate tissue sections with Pretreat $\mathbf{1}$ for $10 \mathrm{~min}$ at $\mathrm{RT}$ for quenching of endogenous peroxidase activity, rinse in $\mathrm{dH}_{2} \mathrm{O}$.

2. Incubate tissue sections with Pretreat 2 maintained at a boiling temperature for 15 min for RNA retrieval, rinse twice in $d \mathrm{H}_{2} \mathrm{O}$.

3. Incubate tissue sections with Pretreat 3 for 30 min at $40^{\circ} \mathrm{C}$ for protein digestion in $\mathrm{HybEZ}$ oven, rinse twice in $\mathrm{dH}_{2} \mathrm{O}$.

\section{Target Probe Hybridization}

Target probes HPV-HR 7 pool include: HPV16, 18, 31, 33, 35, 52, and 58. Add HPV probes, Ubiquitin C (UBC) and bacterial gene dapB probes separately onto three adjacently tissue sections. Hybridize at $40{ }^{\circ} \mathrm{C}$ in oven for $2 \mathrm{hr}$, then rinse in $1 \times$ Wash buffer for $2 \times 2 \mathrm{~min}$ at RT.

\section{Signal Amplification}

1. Incubate tissue sections with Amp1 (preamplifier) for $30 \mathrm{~min}$ at $40^{\circ} \mathrm{C}$, Amp2 (background reducer) for $15 \mathrm{~min}$ at $40{ }^{\circ} \mathrm{C}$, Amp3 (amplifier) for $30 \mathrm{~min}$ at $40{ }^{\circ} \mathrm{C}$, and Amp4 (label probe) for $15 \mathrm{~min}$ at $40^{\circ} \mathrm{C}$ in HybEZ oven. After each hybridization step, rinse in $1 \times$ Wash buffer for $2 \times 2$ $\min$ at RT.

2. Incubate tissue sections with Amp5 for $30 \mathrm{~min}$ and Amp6 for $15 \mathrm{~min}$ at RT. After each incubation step, rinse in $1 \times$ Wash buffer for $2 \times 2$ min at RT.

\section{Signal Detection}

Incubate tissue sections with 1:1 DAB Mixture by mixing equal volume of Brown-A and Brown-B for 10 min at $\mathrm{RT}$, rinse twice in $\mathrm{dH} \mathrm{H}_{2} \mathrm{O}$.

\section{Counterstaining}

Stain tissue sections with $50 \%$ Hematoxylin solution for 2 min at $\mathrm{RT}$, rinse with $\mathrm{dH}_{2} \mathrm{O}$ until slides are clear while tissue remain purple. Dip slides into $0.01 \%$ ammonia in $\mathrm{dH}_{2} \mathrm{O}$ for $5 \mathrm{x}$ and followed with 5 dips in $\mathrm{dH}_{2} \mathrm{O}$.

\section{Slide Mounting}

Dehydrate tissue sections in $70 \%, 100 \%$, and $100 \%$ EtOH for 2 min each, xylene for 5 min, mount with xylene-based mounting media.

\section{Representative Results}

\section{RNAscope HPV assay workflow}

The RNAscope assay has a highly streamlined workflow that is similar to IHC (Figure 1). It consists of four major steps: pretreatments, hybridization, signal amplifications, and detection. It employs a unique probe design strategy that ensures high fidelity signal amplification ${ }^{5}$. In the RNAscope HPV assay, the seven HR-HPV probe sets are pooled, all recognized by the same signal amplification system linked to horseradish peroxidase (HRP). Specific hybridization signals are detected as brown precipitates formed by HRP catalyzed chromogenic reaction using DAB as substrate, which can be readily visualized by standard bright field microscopy. 


\section{Representative staining for HPV detection}

Figure 2 shows example images from stained FFPE sections of head and neck squamous cell carcinoma. In the HPV-positive case (Figure 2A), the HR-HPV probes detected strong punctate signals specifically in the tumor cells. The UBC probe detected numerous punctate cytoplasmic signals in both tumor cells and stromal cells (Figure 2B). The bacterial gene dapB probe demonstrated a clean background (Figure 2C). In the HPV-negative case, both the HR-HPV probe and the dapB probe detected no signals (Figures 2D and 2F), whereas strong signals were detected using the UBC probe (Figure 2E). In this assay, UBC serves as positive control to assess tissue RNA quality and dapB as negative control for background signals. The scoring for HPV status determination involves examining all three slides for each case. The staining level on the negative control slide (dapB) slide is used as cutoff: HPV positivity is defined by the presence of punctate cytoplasmic and/or nuclear staining that was above the signal on the dabpB slide.

\section{RNAscope Flowchart}

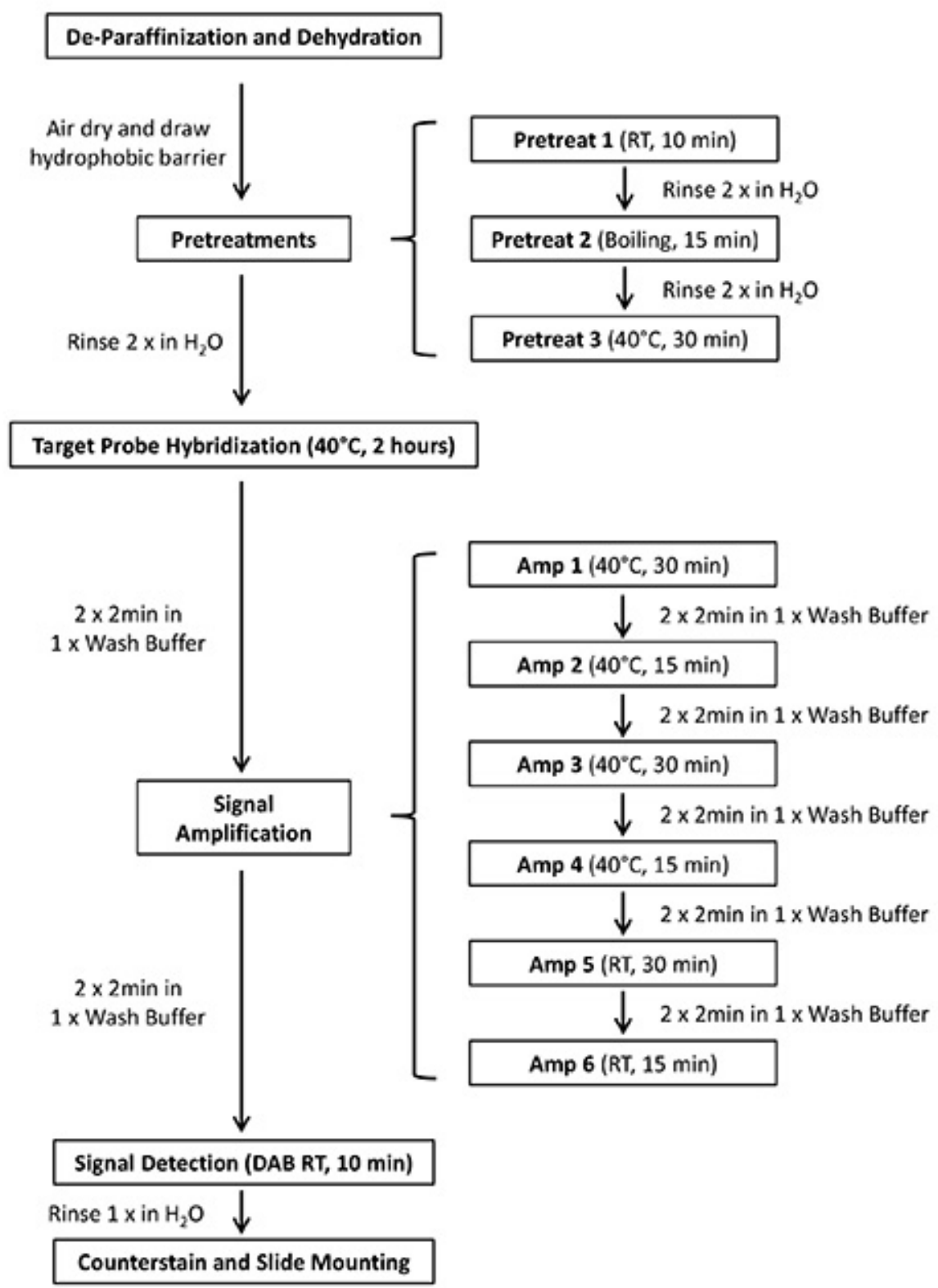

Figure 1. Flowchart of RNAscope assay. Illustration of RNAscope assay workflow. The RNAscope assay contains 4 major steps, pretreatments, target probe hybridization, signal amplification and signal detection. The entire assay procedure can be completed in $8 \mathrm{hr}$. Click here to view larger image. 

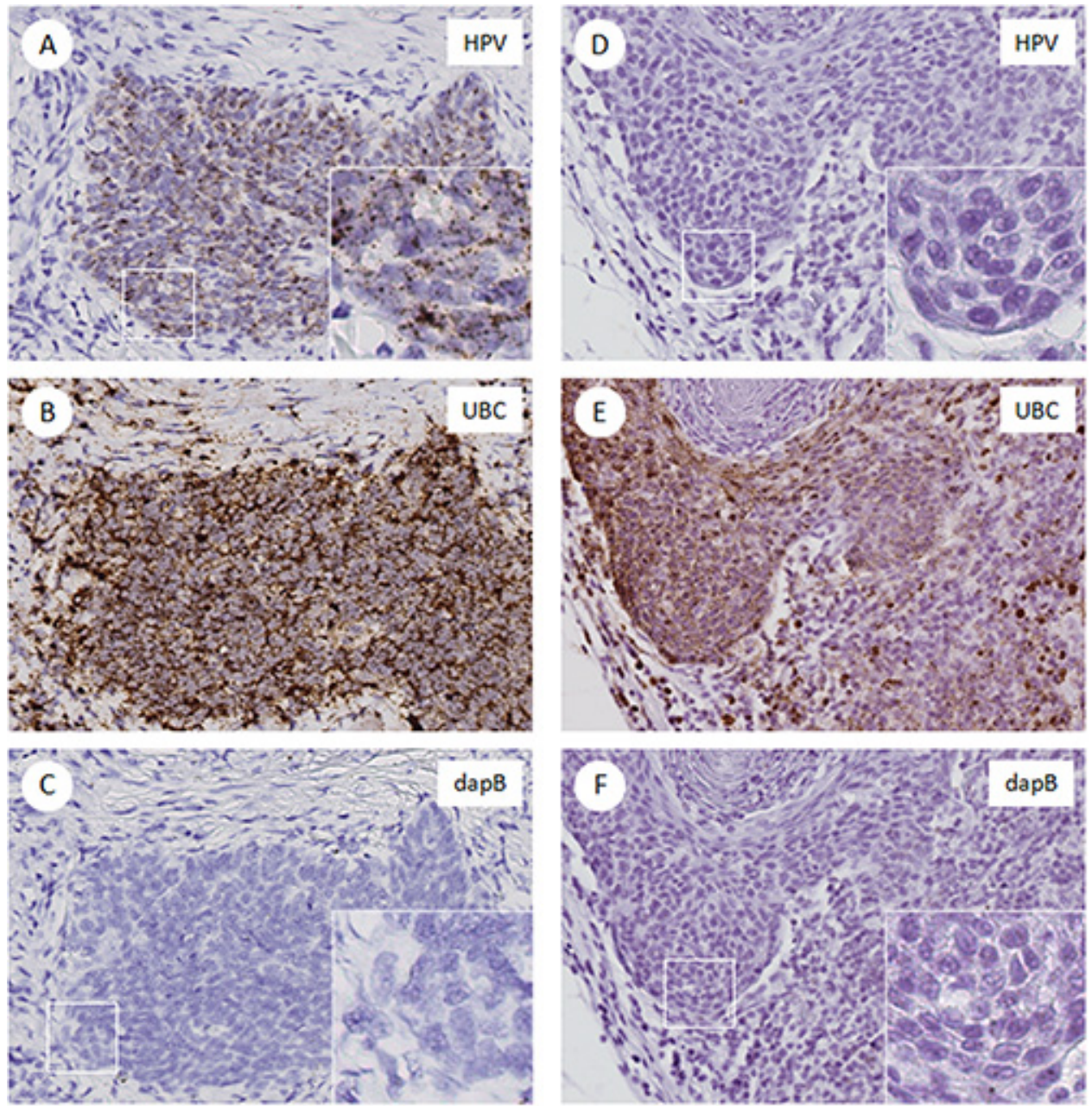

Figure 2. Example images of RNAscope-stained slides. FFPE sections stained with probes for HR-HPV, UBC (positive control) and dapB (negative control) from two HNSCC cases. A-C. HPV-positive case. A, HR-HPV E6/E7 mRNA expression in tumor cells. Inset, 40X magnification to show punctate signals. B and $\mathbf{C}$ ) adjacent tissue sections showing positive UBC staining (B) and negative for dapB (C), respectively. DF) HPV-negative case. D) no staining for HPV E6/E7 mRNA, similar to the dapB negative control (F). E) UBC positive staining. Click here to view larger image.

\section{Discussion}

The RNAscope HPV assay allowed direct visualization of E6/E7 mRNA in situ in HPV-associated head and neck squamous cell carcinoma. The RNAscope assay is fully compatible with routinely fixed tumor tissue and preserves tissue morphology for histopathological correlations (Figure 2). A key advantage of the RNAscope assay over conventional $\mathrm{CISH}$ methods is that it specifically amplifies the hybridization signals (Figures 2B and $2 \mathrm{E}$ ) without amplifying the background noise (Figures $2 \mathrm{C}$ and $2 \mathrm{~F}$ ).

In practice, the RNAscope HPV assay procedure can be completed within $8 \mathrm{hr}$ or conveniently divided over two days. The RNAscope HPV assay has been used to determine HPV status in head and neck squamous cell carcinoma ${ }^{12-16}$, demonstrating $97 \%$ sensitivity and $93 \%$ specificity using qRT-PCR as the reference method ${ }^{16}$. Conventional chromogenic ISH for HR-HPV DNA is highly specific but has a sensitivity of $\sim 80 \%{ }^{12}$ Immunohistochemical $(\mathrm{IHC})$ staining for the cellular surrogate marker p16 demonstrates excellent sensitivity but may generate false positive results $^{15,18}$, especially in nonoropharyngeal head and neck cancers ${ }^{15}$. The current "gold standard" method of qRT-PCR for HPV E6/E7 mRNA detection requires fresh frozen tissue for optimal results and is technically complex, which limits its use to the research laboratory only. In addition, it requires RNA extraction which makes it impossible to correlate HR-HPV E6/E7 mRNA expression with histopathology.

There are several critical factors for the success of the RNAscope assay. First, for best results, tissues should be fixed in fresh $10 \%$ neutralbuffered formalin at room temperature for $16-32 \mathrm{hr}$ according to ASCO/CAP guidelines ${ }^{19}$. Second, the HybEZ oven is highly recommended since it enables optimal control of temperature and humidity for probe hybridization and signal amplification steps. Thirdly, it is important to remove excess residual buffers before each step but still keep the tissue section from drying during any of these steps.

The manual RNAscope procedure described here has been fully automated on a commercial slide auto-staining system ${ }^{10}$. This should greatly facilitate standardization of assay conditions and saving precious manual labor in clinical pathology laboratories. In addition, dedicated image analysis software has been developed ${ }^{10}$ to automatically identify the cells and staining signals on a digitalized slide, which should help to eliminate subjectivity and improve reproducibility in scoring. 
In summary, the RNAscope HPV assay detects the presence of HR-HPV E6/E7 mRNA transcripts in situ in FFPE tissues. It has a workflow that is familiar to clinical pathology laboratories by permitting the directly visualization of these in tissue sections. It offers an ideal platform for examining (FFPE) tissue samples, and can be easily adopted by diagnostic pathology laboratories.

\section{Disclosures}

All authors are employed by and own stock in Advanced Cell Diagnostics, Inc.

Acknowledgements

Supported in part by NIH grant (R43/44CA122444) and DOD BRCP grant (W81XWH-06-1-0682) to YL.

\section{References}

1. Parkin DM. The global health burden of infection-associated cancers in the year 2002. Int. J. Cancer. 118(12), 3030-3044, doi:10.1002/ ijc.21731 (2006).

2. Chaturvedi AK, et al. Human papillomavirus and rising oropharyngeal cancer incidence in the United States. J. Clin. Oncol. 29(32), 4294-4301, doi: 10.1200/JCO.2011.36.4596 (2011).

3. Ang KK, et al. Human papillomavirus and survival of patients with oropharyngeal cancer. N. Engl. J. Med. 363(1), 24-35, doi: 10.1056/ NEJMoa0912217 (2010).

4. Smeets SJ, et al. A novel algorithm for reliable detection of human papillomavirus in paraffin embedded head and neck cancer specimen. Int. J. Cancer. 121(11), 2465-2472, doj: 10.1002/ijc.22980 (2007).

5. Wang F, et al. RNAscope: a novel in situ RNA analysis platform for formalin-fixed, paraffin-embedded tissues. J. Mol. Diagn. 14(1), 22-29, doi: 10.1016/j.jmoldx.2011.08.002 (2012).

6. Liu X, et al. Specific regulation of NRG1 isoform expression by neuronal activity. J. Neurosci. 31(23), 8491-8501, doi: 10.1523/ JNEUROSCI.5317-10.2011 (2011).

7. Yan KS, et al. The intestinal stem cell markers Bmi1 and Lgr5 identify two functionally distinct populations. Proc. Natl. Acad. Sci. U.S.A. 109(2), 466-471, doi: 10.1073/pnas.1118857109 (2012).

8. Payne RE, et al. Viable circulating tumour cell detection using multiplex RNA in situ hybridisation predicts progression-free survival in metastatic breast cancer patients. Br. J. Cancer. 106(11), 1790-1797, doi: 10.1038/bjc.2012.137 (2012).

9. Bordeaux JM, et al. Quantitative in situ measurement of estrogen receptor mRNA predicts response to tamoxifen. PLoS One. 7(5), e36559, doi: 10.1371/journal.pone.0036559 (2012).

10. Wang Z, et al. Automated quantitative RNA in situ hybridization for resolution of equivocal and heterogeneous ERBB2 (HER2) status in invasive breast carcinoma. J. Mol. Diagn. 15(2), 210-219, doi: 10.1016/j.jmoldx.2012.10.003 (2013).

11. Tubbs RR, et al. Ultrasensitive RNA in situ Hybridization for Detection of Restricted Clonal Expression of Low Abundance Immunoglobulin Light Chain mRNA in B-Cell Lymphoproliferative Disorders. Am. J. Surg. Pathol. In press (2013).

12. Ukpo OC, Flanagan JJ, Ma XJ, Luo Y, Thorstad WL, Lewis JS Jr. High-risk human papillomavirus E6/E7 mRNA detection by a novel in situ hybridization assay strongly correlates with $\mathrm{p} 16$ expression and patient outcomes in oropharyngeal squamous cell carcinoma. Am. J. Surg. Pathol. 35(9), 1343-1350, doi: 10.1097/PAS.0b013e318220e59d (2011).

13. Lewis JS Jr, et al. Transcriptionally-active high-risk human papillomavirus is rare in oral cavity and laryngeal/hypopharyngeal squamous cell carcinomas--a tissue microarray study utilizing E6/E7 mRNA in situ hybridization. Histopathology. 60(6), 982-991, doi: 10.1111/ j.1365-2559.2011.04169.x (2012).

14. Lewis JS Jr, et al. Partial p16 staining in oropharyngeal squamous cell carcinoma: extent and pattern correlate with human papillomavirus RNA status. Mod. Pathol. 25(9), 1212-1220, doi: 10.1038/modpathol.2012.79 (2012).

15. Bishop JA, et al. Detection of transcriptionally active high-risk HPV in patients with head and neck squamous cell carcinoma as visualized by a novel E6/E7 mRNA in situ hybridization method. Am. J. Surg. Pathol. 36(12), 1874-1882, doi: 10.1097/PAS.0b013e318265fb2b (2012).

16. Schache AG, et al. Validation of a novel diagnostic standard in HPV-positive oropharyngeal squamous cell carcinoma. Br. J. Cancer. 108(6), 1332-1339, doi: 10.1038/bjc.2013.63 (2013).

17. Mehrad M, et al. Papillary Squamous Cell Carcinoma of the Head and Neck: Clinicopathologic and Molecular Features With Special Reference to Human Papillomavirus. Am. J. Surg. Pathol. Jun 20. [Epub ahead of print], doj: 10.1097/PAS.0b013e318290427d (2013).

18. Jordan, R. C. et al. Validation of methods for oropharyngeal cancer HPV status determination in US cooperative group trials. Am. J. Surg. Pathol. 36, 945-954. doi: 10.1097/PAS.0b013e318253a2d1 (2012).

19. Hammond ME, et al. American Society of Clinical Oncology/College of American Pathologists guideline recommendations for immunohistochemical testing of estrogen and progesterone receptors in breast cancer (unabridged version). Arch. Pathol. Lab. Med. 134(7), e48-72, doi: 10.1043/1543-2165-134.7.e48 (2010). 